\title{
AN INITIALLY DEFORMED FLAT FRAME FINITE ELEMENT
}

\author{
J. ZAMOROWSKI ${ }^{1}$
}

\begin{abstract}
The paper presents the author's non-linear FEM solution of an initially stressless deformed flat frame element, in which the nodes are situated along the axis of the bar initially straight. It has been assumed that each node may sustain arbitrary displacements and rotation. The solution takes into account the effect of shear, the geometrical non-linearity with large displacements (Green-Lagrange's strain tensor) and moderate rotations (i.e. such ones which allow a linear-elastic behaviour of the material) and alternative small rotations when the second Piola-Kirchhoff stress tensor is applied. This solution is based on [1], concerning beams without any initial bow imperfections. The convergence of the obtained results at different numbers of nodes and Gauss points in the element was tested basing on the example of circular arcs with a central angle of $120^{\circ} \div 180^{\circ}$. The analysis concerned elements with two, three, five, seven, nine and eleven nodes, for the same number of points of numerical integration and also with one more or less. Moreover, the effect of distributing the load on the convergence of the results was analyzed.
\end{abstract}

Keywords: initially deformed flat frame element, geometrical non-linearity, large displacements, large and small rotations.

\section{INTRODUCTION}

The existing FEM solutions, quoted for instance in i.e. [1-17] describe rather well the geometrically non-linear behaviour of straight bars, frame bars, or regularly curved bars i.e. Litewka [8], Lenci [9], Pai [10], Rosen [12], but they are not always satisfactory in the case of initially deformed beams and frame elements with an arbitrary field of initiall deformations. Computational models of curved elements consist mainly of circular curves, parabolic or any other number of axes defined as smooth curves.

Most theories dealing with that problem since the " 60 s of the past century contain some simplifications concerning, e.g. smaller displacements, small normal strains or tangents - see e.g. Rosen [12] and Tang [13], small rotations (i.e. Zienkiewicz [14]), a small step of loading and usually the lack of initial deformations. By increasing the increment of the load during the calculations some of the assumptions may be easily exceeded, and then the results of the calculations will prove to be inaccurate.

1 Silesian University of Technology in Gliwice, Faculty of Civil Engineering, 5 Akademicka Street, 44-100 Gliwice, Poland, Zamski@interia.pl 
Solutions concerning straight and curved elements are based on the theory of the Bernoully beam (e.g. Lenci [9]), where the effect of shear are left out of account or on the theory of Timoshenko's beam - see e.g. Mingrui Li [1], Rakowski [11], Gołaś [16], Zamorowski [18], where this effect was not been neglected.

The model of a one-dimensional initially bent, heterogeneous beam, presented by Lenci in [9], was achieved by making use of the three-dimensional (3D) mechanics of continuous media. The kinetics of the element was described by four one dimensional functions indicating radial, tangent displacements as well as the displacement from the plane of the beam, caused by its bending and torsion and the angular displacement of the cross-section of the beam.

Tang presents in his paper [13] the non-linear behaviour of naturally bent and twisted beams, taking into account the effect of shear in the case of large displacements and rotations but only small strains. More accurate beam element with imperfections at a large axial load was dealt with by Adman [19]. Preliminary imperfections were replaced by a sinusoidal initial bending of the beam. Shape functions were obtained by means of a differential equation of the deformed axis of the bar. The bars were modelled by only one element, which proved to be favourable with respect to the dimensions of the matrix of rigidity of the whole structure.

Mingrui Li in [1] presents a geometrically nonlinear theory of a 2-D beam in which large displacements, large rotations and the effect of shear are taken into account. This theory was developed with only one simplification, assuming that Poisson's coefficient $v=0$, which means that $E=2 G$. This theory is correct both in the case of a straight and a curved beam. The difference consists in the fact that in the case of a straight beam the local arrangement of co-ordinate systems may be determined as a global system of the whole element, while in the curved beam this arrangement ought to be determined for each point individually. Practically, while calculating curved elements the arrangement is determined in every point of numerical integration. The results obtained in compliance with such a solution are equally accurate both in the case of distributing the load on any given number of steps and without this distribution. According to the solutions, e.g. Mingrui Li [1] or Lenci [9], the matrices of the transformation of displacements from local systems to a global system in straight beams may be excluded in front of the sign of the integral, whereas in curved beams these matrices must remain under the sign of the integral, which prolongs the time of calculations.

The literature provides too few exact solutions concerning steel structures of bars with arbitrary geometrical imperfections, which are in the case of global imperfections related to the theoretical position of the nodes in the structure, and in the case of local imperfections (along the bar) they are measured in relation to the chord spreading between the existing nodes of the deformed bar.

The present paper contains the author's nonlinear FEM solution concerning a flat frame element with an arbitrary field of initially stressless deformations (displacements and rotation), taking into consideration the shearing as in Timoshenko's beam. The element with nodes placed along a straight line, and an initial deformation is described 
using translations and rotations of the nodes. This solution includes the complete description of Lagrange, the so-called Total Lagrangian [TL] with Green-Lagrange's strain tensor and the second Piola-Kirchhoff stress tensor. This solution is based on the work of Mingrui Li [1], which deals with bars without initial deformation. An elastic material model is assumed, including a large displacement and alternatively large or small rotations. This model can be applied in detail analysis of flat bar structures with any geometrical imperfections and structures with tension members, as well as for the purpose of assessing other less accurate solutions.

\section{Geometrical ReLations}

The initial coordinate system $X Y$ was assumed as for the initially not deformed configuration - Fig. la.

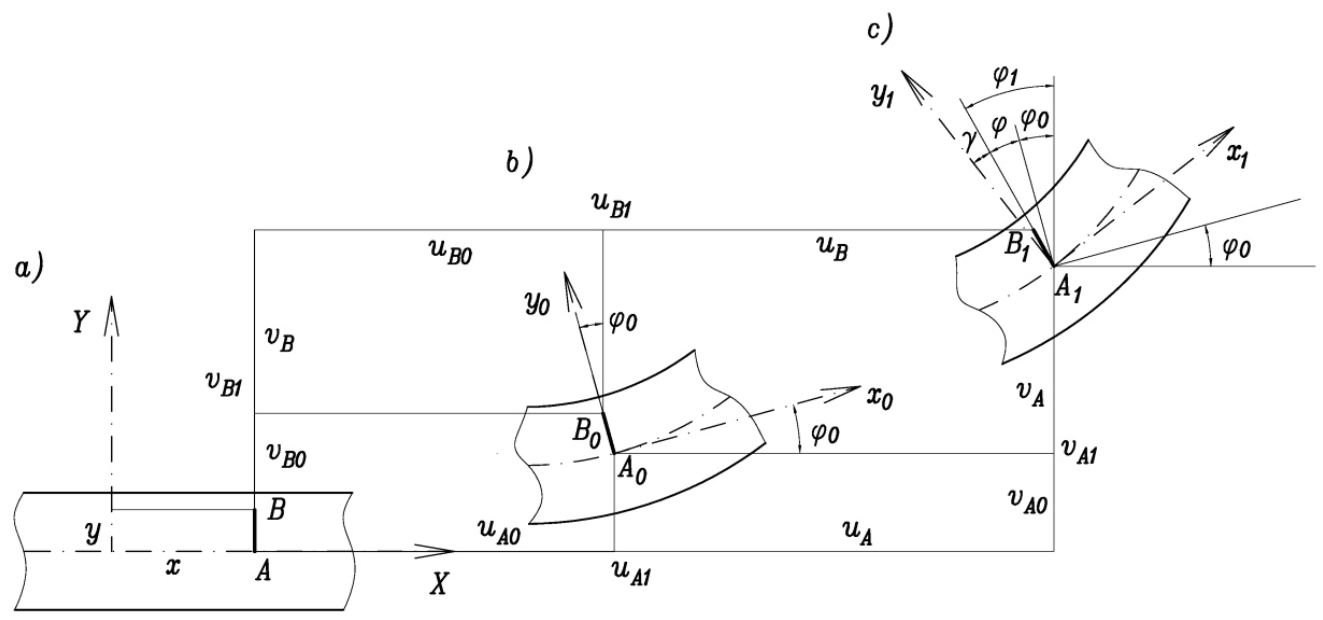

Fig. 1. Configuration of a bar: a) initial, b) initially deformed, c) elastically deformed.

The initial state for the increment of forces and displacements was assumed to be an initially stressles deformed element as shown in Fig, 1b, the arbitrary cross-section of which was initially displaced $\left(u_{A 0}, v_{A 0}\right)$ in relation to an element without any geometrical imperfections, and rotated by the angle $\varphi_{0}$. The effect of shear was taken into account similarly as in the Timoshenko beam, in which the cross-sections initially normal to the neutral axis (Fig. 1a and 1b), do not remain normal to this axis after their deformation (Fig. lc), and the angle of shear is $\gamma$. Considering Green-Lagrange's strain tensor as well as the second Piola-Kirchhoff stress tensor, formulae were derived for large displacements, moderately large rotations (at which a linear-elastic behaviour of the material is still possible) and alternatively also for small rotations. The solution is 
based on the assumption that the length of any arbitrary segment of the cross-section $A B$ remains unchanged after the deformation, which means that Poisson's coefficient $v=0$ is correct.

Displacements of the points $A$ and $B$, situated in the cross-section of the element, which is positioned at the distance $x$ from the beginning of the coordinate system $X, Y$, is considered, assuming that the point $A$ is situated on the neutral axis of the element, and point $B$ at the distance $y$ from this axis. The coordinates of the points $A_{0}$ and $B_{0}$ in the initial system $X, Y$ are after the preliminary stressless deformation of the element, are expressed by the formula

$$
x_{A 0}=x+u_{A 0}, y_{A 0}=0+v_{A 0}, x_{B 0}=x+u_{B 0}, y_{B 0}=y+v_{B 0} .
$$

The coordinates of the points $A_{1}, B_{1}$ after the elastic deformation

$$
x_{A 1}=x_{A 0}+u_{A}, y_{A 1}=y_{A 0}+v_{A}, x_{B 1}=x_{B 0}+u_{B}, y_{B 1}=y_{B 0}+v_{B} \text {. }
$$

The coordinates of the point $B_{0}$, determined by means of the formula (2.1), may also be expressed by the coordinates of the points $A_{0}$ and the angle of preliminary rotation of the cross-section $\varphi_{0}$. In the case of large rotations we have

$$
x+u_{B 0}=x+u_{A 0}-y \sin \varphi_{0}, y+v_{B 0}=0+v_{A 0}+y \cos \varphi_{0} .
$$

Hence, neglecting the index $A$ for a point situated on the neutral axis

$$
u_{B 0}=u_{0}-y \sin \varphi_{0}, v_{B 0}=v_{0}-y+y \cos \varphi_{0}=v_{0}-y\left(1-\cos \varphi_{0}\right) .
$$

Assuming small rotations it may be

$$
\begin{gathered}
\sin \varphi=\varphi, \sin \varphi_{0}=\varphi_{0}, \cos \varphi=\cos \varphi_{0}=1, \\
\sin \left(\varphi_{0}+\varphi\right)=\sin \varphi_{0} \cos \varphi+\cos \varphi_{0} \sin \varphi=\varphi_{0}+\varphi, \cos \left(\varphi_{0}+\varphi\right)=1-\varphi_{0} \varphi
\end{gathered}
$$

as well as

$$
x+u_{B 0}=x+u_{A 0}-y \varphi_{0}, y+v_{B 0}=0+v_{A 0}+y .
$$

Thus, neglecting the index $A$

$$
u_{B 0}=u_{0}-y \varphi_{0}, v_{B 0}=v_{0} .
$$

Analogically, for the point $B_{1}$ we have got:

- in the case of large rotations

$$
\begin{aligned}
& x+u_{B 0}+u_{B}=x+u_{A 0}+u_{A}-y \sin \left(\varphi_{0}+\varphi\right), \\
& y+v_{B 0}+v_{B}=0+v_{A 0}+v_{A}+y \cos \left(\varphi_{0}+\varphi\right) .
\end{aligned}
$$


- in the case of small rotations

$$
\begin{aligned}
& x+u_{B 0}+u_{B}=x+u_{A 0}+u_{A}-y\left(\varphi_{0}+\varphi\right), \\
& y+v_{B 0}+v_{B}=0+v_{A 0}+v_{A}+y\left(1-\varphi_{0} \varphi\right) .
\end{aligned}
$$

Substituting (2.2) and (2.3), respectively, in (2.4) and (2.5) after transformations and neglecting the index $A$ we have:

- in the case of large rotations

$$
u_{B}=u-y \sin \left(\varphi_{0}+\varphi\right)+y \sin \varphi_{0}, v_{B}=v+y \cos \left(\varphi_{0}+\varphi\right)-y \cos \varphi_{0} .
$$

- $\quad$ in the case of small rotations

$$
u_{B}=u-y\left(\varphi_{0}+\varphi\right)+y \varphi_{0}=u-y \varphi, v_{B}=v-y \varphi_{0} \varphi
$$

Green-Lagrange's strain tensor may be determined as the difference of tensors in the deformed and initially deformed state, i.e. Kleiber [20]

$$
E_{i j}=E_{i j}\left(u_{0}+u, v_{0}+v\right)-E_{i j}\left(u_{0}, v_{0}\right), \text { for }\{i, j\}=\{x, y\}
$$

or derived from the definition of the tensor - cf. Zamorowski [18]

$$
2 E_{i j} d a_{i} d a_{j}=d s_{1}^{2}-d s_{0}^{2},
$$

where: $a_{1}, a_{2}, a_{3}-$ the initial system of the coordinates and $d s_{0}$ and $d s_{1}-$ the lengths of an infinitesimal linear element, previous to and after the deformation, respectively.

In the case of the gradient of displacements of the point $B_{1}$, and taking into account the derivatives of the functions (2.2) and (2.6) as well as the relations $u_{B 1}=u_{B 0}+u_{B}$, $v_{B 1}=v_{B 0}+v_{B}$, at large rotations from equation (2.8) is obtained

$$
\begin{gathered}
E_{x x}=\left(1+u_{0 . x}\right) u_{. x}+\left(u_{. x}\right)^{2} / 2+\left(v_{0 . x}+v_{. x} / 2\right) v_{. x}+y^{2}\left(\varphi_{0 . x}+\varphi_{. x} / 2\right) \varphi_{. x} \\
-y\left(\varphi_{0 . x}+\varphi_{. x}\right)\left(1+u_{0 . x}+u_{. x}\right) \cos \left(\varphi_{0}+\varphi\right)-y\left(\varphi_{0 . x}+\varphi_{. x}\right)\left(v_{0 . x}+v_{. x}\right) \sin \left(\varphi_{0}+\varphi\right) \\
+y\left(1+u_{0 . x}\right) \varphi_{0 . x} \cos \varphi_{0}+y v_{0 . x} \varphi_{0 . x} \sin \varphi_{0}, \\
2 E_{x y}=\left(v_{0 . x}+v_{. x}\right) \cos \left(\varphi_{0}+\varphi\right)-\left(1+u_{0 . x}+u_{. x}\right) \sin \left(\varphi_{0}+\varphi\right) \\
+\left(1+u_{0 . x}\right) \sin \varphi_{0}-v_{0 . x} \cos \varphi_{0}, E_{y y}=0 .
\end{gathered}
$$

The components of Green-Lagrange's strain tensor may be expressed in the form of a matrix, which permits to determine the elements of the secant of the matrix of rigidity as follows 


$$
E_{x x}=\mathbf{E}_{x x} \mathbf{e}+\mathbf{E}_{x x}^{0} \mathbf{e}_{0}, 2 E_{x y}=\mathbf{E}_{x y} \mathbf{e}+\mathbf{E}_{x y}^{0} \mathbf{e}_{0}, E_{y y}=\mathbf{0},
$$

where

$$
\mathbf{e}=\left[\begin{array}{llll}
u_{. x} & v_{. x} & \varphi_{. x} & \varphi
\end{array}\right]^{T}, \mathbf{e}_{0}=\left[\begin{array}{llll}
u_{0 . x} & v_{0 . x} & \varphi_{0 . x} & \varphi_{0}
\end{array}\right]^{T},
$$

and

$$
\begin{aligned}
& \mathbf{E}_{x x}=\left[\begin{array}{llll}
E_{x x 1} & E_{x x 2} & E_{x x 3} & 0
\end{array}\right], \mathbf{E}_{x x}^{0}=\left[\begin{array}{llll}
0 & E_{x x 2}^{0} & E_{x x 3}^{0} & 0
\end{array}\right], \\
& \mathbf{E}_{x y}=\left[\begin{array}{lllll}
E_{x y 1} & E_{x y 2} & 0 & E_{x y 4}
\end{array}\right], \mathbf{E}_{x y}^{0}=\left[\begin{array}{llll}
E_{x y 1}^{0} & E_{x y 2}^{0} & 0 & 0
\end{array}\right] .
\end{aligned}
$$

In the case of large rotations

$$
\begin{gathered}
E_{x x 1}=\left(1+u_{0 . x}\right)+u_{. x} / 2-y\left(\varphi_{0 . x}+\varphi_{. x}\right) \cos \left(\varphi_{0}+\varphi\right), \\
E_{x x 2}=\left(v_{0 . x}+v_{. x} / 2\right)-y\left(\varphi_{0 . x}+\varphi_{. x}\right) \sin \left(\varphi_{0}+\varphi\right), \\
E_{x x 3}=y^{2}\left(\varphi_{0 . x}+\varphi_{. x} / 2\right)-y\left(1+u_{0 . x}\right) \cos \left(\varphi_{0}+\varphi\right)-y v_{0 . x} \sin \left(\varphi_{0}+\varphi\right), \\
E_{x x 2}^{0}=y \varphi_{0 . x}\left[\sin \varphi_{0}-\sin \left(\varphi_{0}+\varphi\right)\right], E_{x x 3}^{0}=y\left(1+u_{0 . x}\right)\left[\cos \varphi_{0}-\cos \left(\varphi_{0}+\varphi\right)\right], \\
E_{x y 1}=-\sin \left(\varphi_{0}+\varphi\right), E_{x y 2}=\cos \left(\varphi_{0}+\varphi\right), \\
E_{x y 4}=\left[\sin \varphi_{0}-\sin \left(\varphi_{0}+\varphi\right)\right] / \varphi, \text { when } \varphi=0 \text { then } \\
E_{x y 4}=\left[\sin \varphi_{0}-\sin \left(\varphi_{0}+\varphi\right)\right]_{. \varphi} / \varphi_{. \varphi}=-\cos \left(\varphi_{0}+\varphi\right) / 1=-\cos \varphi_{0}, \\
E_{x y 1}^{0}=\sin \varphi_{0}-\sin \left(\varphi_{0}+\varphi\right), E_{x y 2}^{0}=-\cos \varphi_{0}+\cos \left(\varphi_{0}+\varphi\right) .
\end{gathered}
$$

In the case of small rotations and derivatives of the function (2.3) and (2.7)

$$
\begin{gathered}
E_{x x}=\left[1+u_{0 . x}+u_{. x} / 2-y\left(\varphi_{0 . x}+\varphi_{. x}\right)\right] u_{. x}+\left[v_{0 . x}+v_{. x} / 2-y\left(\varphi_{0 . x} \varphi+\varphi_{0} \varphi_{. x}\right)\right] v_{. x} \\
+\left[y^{2}\left(1+\varphi_{0}^{2}\right) \varphi_{. x} / 2+y^{2} \varphi_{0 . x}\left(1+\varphi_{0} \varphi\right)-y\left(1+u_{0 . x}\right)-y v_{0 . x} \varphi_{0}\right] \varphi_{. x} \\
+y \varphi_{0 . x}\left(y \varphi_{0 . x} \varphi / 2-v_{0 . x}\right) \varphi \\
2 E_{x y}=-\left(\varphi_{0}+\varphi\right) u_{. x}+\left(1-\varphi_{0} \varphi\right) v_{. x}+y \varphi\left(1+\varphi_{0}^{2}\right) \varphi_{. x}-\left[1+u_{0 . x}+\varphi_{0}\left(v_{0 . x}-y \varphi_{0 . x} \varphi\right)\right] \varphi, \\
E_{y y}=\varphi^{2} / 2+\varphi_{0}^{2} \varphi^{2} / 2=\frac{\varphi^{2}}{2}\left(1+\varphi_{0}^{2}\right) .
\end{gathered}
$$

Taking into account (2.9) it has been assumed that $E_{y y}=0$. 


\section{Physical Relations}

In the case of an elastic, homogeneous, isotropic material the physical relation is assumed to take the form of the second Piola-Kirchhoff stress tensor. In compliance with [21]

$$
T_{i j}^{(2)}=\beta_{0} \delta_{i j}+\beta_{1} E_{i j}+\beta_{2} E_{i k} E_{k j} \text {, for }\{i, j\}=\{x, y\} \text { and }\{k\}=\{x, y\} \text {, }
$$

where $E$ is Green-Lagrange's strain tensor and $\beta_{\alpha}$ are functions or constant of material. Introducing $\beta_{0}=\lambda I_{1}^{E}, \beta_{1}=2 \mu, \beta_{2}=0$ into (3.1) where $I_{1}^{E}-$ is the first invariant of the Green-Lagrange's strain tensor and $\lambda, \mu$ - are Lame's physical constants, we get Hooke's linear physical law

$$
T_{i j}^{(2)}=2 \mu E_{i j}+\lambda \delta_{i j} E_{k k} .
$$

Considering in compliance with this assumption that $v=0$ and

$$
\lambda=\frac{v E}{(1+v)(1-2 v)}=0, \mu=\frac{E}{2(1+v)}=\frac{E}{2}=G \text { but } \delta_{i, j}=\left\{\begin{array}{l}
1, i=j \\
0, i \neq j
\end{array},\right.
$$

in the case of a flat state of stresses, when $E_{y y}=0$ (c.f. 2.9), we get

$$
\begin{gathered}
T_{x x}^{(2)}=2 \mu E_{x x}=E E_{x x}, \\
T_{y y}^{(2)}=2 \mu E_{y y}=0, \\
T_{x y}^{(2)}=2 \mu E_{x y}=G \cdot 2 E_{x y} .
\end{gathered}
$$

\section{Shape FUnCtions AND INTERPOLATION FUNCTIONS OF DISPLACEMENTS}

In the case of curved elements the proper choice of the shape function is of an essential importance. Depending on the applied functions various results may be obtained, particularly when the height of the cross-sections is small in relation to the radius of the curvature of the bar. This phenomenon was perceived already in the seventh decade of the past century in arches and called shear locking. It has been found that it occurs when the field of displacements is approximated by means of polynomials of a low order, particularly linear and square shape functions applied in two- and three-node elements. Later, an avoiding of the effect of this phenomenon was suggested on various ways. The number of points of numerical integration concerning some components of elastic strain energy was reduced. In other investigations, e.g. Litewka [8], trigonometric shape functions or polynomial functions were applied, which are an approximation of exact trigonometric functions. Jelenić [6] proposed to interpolate the displacements by means of the hierarchic function of polynomials. This formulation was also used based on co-rotation [22]. 
In the present paper, the shape functions were applied in the form of Lagrange's polynomials of a higher-order [23]. The suitability of elements with two, three, five, seven, nine and eleven nodes was analyzed. The results of calculations, based on solutions presented in the paper, indicate that displacements of a bar initially deformed to the shape of an arc converge with the same values, independently of the number of components of the polynomial, if polynomials of a higher-order are applied than a linear one. This was checked by applying elements with three up to nine nodes with shape functions in the form of polynomials of the second to the eighth order. In the case of two-node elements (with a linear shape function) the process of convergence was very slow. Satisfactory results were not achieved even when the arc was divided into 1000 elements. The convergence was somewhat faster when elements with three nodes were applied. Then a division into about 250 elements was sufficient. A considerable accuracy of results was achieved in the case of elements with five nodes, when the arc was divided into 18 elements, in the case of elements with seven nodes divided into 8 elements and in the case of nine-node elements into 5 elements. In all these cases in the calculations the number of Gauss points was assumed to equalize the number of nodes in the elements.

The shape function was assumed as for natural coordinates. In the case of a growing numeration of nodes (Fig. 2) we get

$$
N_{i}=\prod_{j=1 ; j \neq i}^{n} \frac{\xi-\xi_{j}}{\xi_{i}-\xi_{j}}, \text { where } \xi_{i}=-1+\frac{2(i-1)}{n-1} .
$$
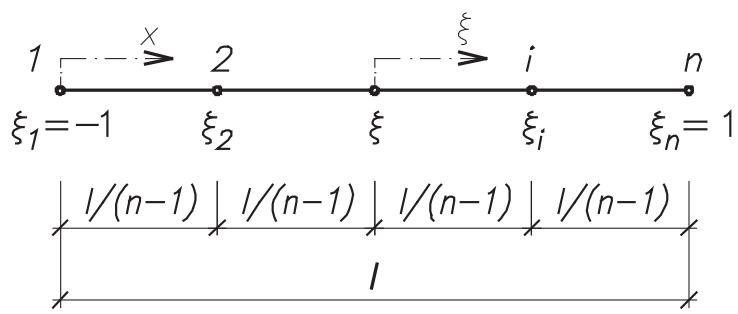

Fig. 2. Element in natural coordinates

The displacements of the element and the geometry are expressed by the same interpolation functions

$$
x=\sum_{i=1}^{n} N_{i} x_{i}
$$

$$
u_{0}=\sum_{i=1}^{n} N_{i} u_{0 i}, v_{0}=\sum_{i=1}^{n} N_{i} v_{0 i}, \varphi_{0}=\sum_{i=1}^{n} N_{i} \varphi_{0 i}, u=\sum_{i=1}^{n} N_{i} u_{i}, v=\sum_{i=1}^{n} N_{i} v_{i}, \varphi=\sum_{i=1}^{n} N_{i} \varphi_{i},
$$


where: $x_{i}$-coordinates of the nodal points, $n$-number of nodal points in the element, $u_{0}, v_{0}, \varphi_{0}$ - preliminary stressless displacements, $u, v, \varphi$ - elastic displacements,

$u_{0 i}, v_{0 i}, \varphi_{0 i}$ and $u_{i}, v_{i}, \varphi_{i}-$ preliminary and elastic displacements of the nodal points.

Substituting into expression (4.1) shape functions with the corresponding nodal coordinates $x_{i}$, with any number of nodes, we get the relation

$$
x=(1+\xi) l / 2 \text { and } \xi=2 x / l-1 .
$$

The components of strains of the element are expressed, among others, by derivatives of displacements in relation to the Cartesian coordinates, and the displacements are expressed by shape functions in natural coordinates; the derivatives of the shape function ought to be also determined with respect to Cartesian coordinates.

Then we have

$$
N_{i . x}=N_{i . \xi} \cdot \xi_{. x}=\frac{2}{l} N_{i . \xi} \text { but } N_{i . \xi}=\sum_{\substack{k=1 \\ k \neq i}}^{n}\left[\prod_{j=1 ; j \neq i, j \neq k}^{n}\left(\xi-\xi_{j}\right) / \prod_{j=1 ; j \neq i}^{n}\left(\xi_{i}-\xi_{j}\right)\right],
$$

where $2 / l=\mathbf{J}^{-1}$ is the reciprocal of the Jacobi matrix concerning the bar element.

Introducing selective functions (interpolation functions for displacements)

$$
\mathbf{H}=\left[\begin{array}{llllll}
\mathbf{H}_{1} & \mathbf{H}_{2} & . . & \mathbf{H}_{i} & . . & \mathbf{H}_{n}
\end{array}\right],
$$

where

$$
\mathbf{H}_{i}=\left[\begin{array}{ccc}
N_{i . x} & 0 & 0 \\
0 & N_{i . x} & 0 \\
0 & 0 & N_{i . x} \\
0 & 0 & N_{i}
\end{array}\right]=\frac{2}{l}\left[\begin{array}{ccc}
N_{i . \xi} & 0 & 0 \\
0 & N_{i . \xi} & 0 \\
0 & 0 & N_{i . \xi} \\
0 & 0 & \frac{l}{2} N_{i}
\end{array}\right],
$$

we may write

$$
\mathbf{e}=\mathbf{H q}, \mathbf{e}_{0}=\mathbf{H q} \mathbf{q}_{0},
$$

where $\mathbf{e}, \mathbf{e}_{0}$ according to (2.11) and displacements of the nodal points

$$
\begin{aligned}
\mathbf{q}=\left[\begin{array}{llllll}
\mathbf{q}_{1} & \mathbf{q}_{2} & . . & \mathbf{q}_{i} & . . & \mathbf{q}_{n}
\end{array}\right]^{T}, \mathbf{q}_{0}=\left[\begin{array}{llllll}
\mathbf{q}_{01} & \mathbf{q}_{02} & . . & \mathbf{q}_{0 i} & . . & \mathbf{q}_{0 n}
\end{array}\right]^{T}, \\
\mathbf{q}_{i}=\left[\begin{array}{llll}
u_{i} & v_{i} & \varphi_{i}
\end{array}\right]^{T}, \mathbf{q}_{0 i}=\left[\begin{array}{llll}
u_{0 i} & v_{0 i} & \varphi_{0 i}
\end{array}\right]^{T} .
\end{aligned}
$$




\section{INCREASING EQUATIONS OF EQUILIBRIUM}

Equations of equilibrium of the element are based on the principle of virtual work

$$
\delta L_{z}=\delta L_{w},
$$

where $\delta L_{z}, \delta L_{w}$ - virtual work of external and internal forces.

Introducing an equivalent nodal load $P_{i}$ instead of an arbitrary internodal load $p$ the virtual work of external forces may be expressed as

$$
\delta L_{z}=\delta \mathbf{q}^{T} \mathbf{P} \text {, where } \mathbf{P}=\left[\begin{array}{llllll}
\mathbf{P}_{1} & \mathbf{P}_{2} & . . & \mathbf{P}_{i} & . . & \mathbf{P}_{n}
\end{array}\right]^{T} \text { and } \mathbf{P}_{i}=\left[\begin{array}{lll}
P_{i x} & P_{i y} & M_{i}
\end{array}\right]^{T} .
$$

The virtual work of internal forces in the primary configuration is obtained from the expression

$$
\delta L_{w}=\int_{V} \delta E^{T} T^{(2)} d V
$$

in which the components of Green-Lagrange's strain tensor $(E)$ and the second Piola-Kirchhoff stress tensor $\left(T^{(2)}\right)$ are coupled energy values, and $V$ is the primary volume of the material.

Taking into account (3.2), we get

$$
\delta L_{w}=\int_{V}\left[\delta E_{x x}^{T} T_{x x}^{(2)}+\delta\left(2 E_{x y}\right)^{T} T_{x y}^{(2)}\right] d V=\int_{V}\left[\delta E_{x x}^{T} E E_{x x}+\delta\left(2 E_{x y}\right)^{T} \cdot G\left(2 E_{x y}\right)\right] d V .
$$

Introducing variants of the components of Green-Lagrange's tensor in the form of

$$
\delta E_{x x}=\mathbf{A}_{x x} \delta \mathbf{e}=\mathbf{A}_{x x} \mathbf{H} \delta \mathbf{q}=\mathbf{B}_{x x} \delta \mathbf{q}, \delta\left(2 E_{x y}\right)=\mathbf{A}_{x y} \delta \mathbf{e}=\mathbf{A}_{x y} \mathbf{H} \delta \mathbf{q}=\mathbf{B}_{x y} \delta \mathbf{q},
$$

where in the case of large rotations

$$
\begin{aligned}
& \mathbf{A}_{x x}=\left[\begin{array}{llll}
A_{1 x x} & A_{2 x x} & A_{3 x x} & A_{4 x x}
\end{array}\right], \mathbf{A}_{x y}=\left[\begin{array}{llll}
A_{1 x y} & A_{2 x y} & A_{3 x y} & A_{4 x y}
\end{array}\right], \\
& A_{1 x x}=1+u_{0 . x}+u_{. x}-y\left(\varphi_{0 . x}+\varphi_{. x}\right) \cos \left(\varphi_{0}+\varphi\right), \\
& A_{2 x x}=v_{. x}+v_{0 . x}-y\left(\varphi_{0 . x}+\varphi_{. x}\right) \sin \left(\varphi_{0}+\varphi\right) \text {, } \\
& A_{3 x x}=y\left[y\left(\varphi_{0 . x}+\varphi_{. x}\right)-\left(1+u_{0 . x}+u_{. x}\right) \cos \left(\varphi_{0}+\varphi\right)-\left(v_{0 . x}+v_{. x}\right) \sin \left(\varphi_{0}+\varphi\right)\right] \text {, } \\
& A_{4 x x}=y\left(\varphi_{0 . x}+\varphi_{. x}\right)\left[\left(1+u_{0 . x}+u_{. x}\right) \sin \left(\varphi_{0}+\varphi\right)-\left(v_{0 . x}+v_{. x}\right) \cos \left(\varphi_{0}+\varphi\right)\right] \text {, } \\
& A_{1 x y}=-\sin \left(\varphi_{0}+\varphi\right), A_{2 x y}=\cos \left(\varphi_{0}+\varphi\right), A_{3 x y}=0, \\
& A_{4 x y}=-\left(1+u_{0 . x}+u_{. x}\right) \cos \left(\varphi_{0}+\varphi\right)-\left(v_{0 . x}+v_{. x}\right) \sin \left(\varphi_{0}+\varphi\right) \text {, }
\end{aligned}
$$

we get the matrix equation of equilibrium of the element 


$$
\psi(\mathbf{q})=\int_{V}\left(\mathbf{B}_{x x}^{T} T_{x x}^{(2)}+\mathbf{B}_{x y}^{T} T_{x y}^{(2)}\right) d V-\mathbf{P}=0 .
$$

In the case of small rotations

$$
\begin{gathered}
A_{1 x x}=1+u_{0 . x}+u_{. x}-y\left(\varphi_{0 . x}+\varphi_{. x}\right) \cos \left(\varphi_{0}+\varphi\right), \\
A_{2 x x}=v_{. x}+v_{0 . x}-y\left(\varphi_{0 . x}+\varphi_{. x}\right) \sin \left(\varphi_{0}+\varphi\right), \\
A_{3 x x}=y\left[y\left(\varphi_{0 . x}+\varphi_{. x}\right)-\left(1+u_{0 . x}+u_{. x}\right) \cos \left(\varphi_{0}+\varphi\right)-\left(v_{0 . x}+v_{. x}\right) \sin \left(\varphi_{0}+\varphi\right)\right], \\
A_{4 x x}=y\left(\varphi_{0 . x}+\varphi_{. x}\right)\left[\left(1+u_{0 . x}+u_{. x}\right) \sin \left(\varphi_{0}+\varphi\right)-\left(v_{0 . x}+v_{. x}\right) \cos \left(\varphi_{0}+\varphi\right)\right], \\
A_{1 x y}=-\sin \left(\varphi_{0}+\varphi\right), A_{2 x y}=\cos \left(\varphi_{0}+\varphi\right), A_{3 x y}=0, \\
A_{4 x y}=-\left(1+u_{0 . x}+u_{. x}\right) \cos \left(\varphi_{0}+\varphi\right)-\left(v_{0 . x}+v_{. x}\right) \sin \left(\varphi_{0}+\varphi\right),
\end{gathered}
$$

A solution in the increasing form may be obtained from the equation (5.1) after its development into Taylor's series. Confining ourselves to only the linear part of this development we get

$$
\psi(\mathbf{q}+\Delta \mathbf{q})=\psi(\mathbf{q})+\frac{\partial \psi(\mathbf{q})}{\partial \mathbf{q}} \Delta \mathbf{q}+O(\Delta \mathbf{q})^{2}=\mathbf{0},
$$

hence

$$
\frac{\partial \psi(\mathbf{q})}{\partial \mathbf{q}} \Delta \mathbf{q}=\mathbf{K}_{T} \Delta \mathbf{q}=-\psi(\mathbf{q})=\mathbf{P}-\int_{V}\left(\mathbf{B}_{x x}^{T} T_{x x}^{(2)}+\mathbf{B}_{x y}^{T} T_{x y}^{(2)}\right) d V .
$$

A tangent matrix may be got from (5.1)

$$
\mathbf{K}_{T}=\frac{\partial \psi(\mathbf{q})}{\partial \mathbf{q}}=\frac{\partial \psi(\mathbf{q})}{\partial \mathbf{e}} \mathbf{H} .
$$

Taking into account

$$
\begin{aligned}
& \frac{\partial\left(\mathbf{B}_{x x}^{T} T_{x x}^{(2)}\right)}{\partial \mathbf{e}}=\frac{\partial \mathbf{B}_{x x}^{T}}{\partial \mathbf{e}} T_{x x}^{(2)}+\mathbf{B}_{x x}^{T} \frac{\partial T_{x x}^{(2)}}{\partial \mathbf{e}}=\mathbf{H}^{T} \frac{\partial \mathbf{A}_{x x}^{T}}{\partial \mathbf{e}} T_{x x}^{(2)}+E \mathbf{B}_{x x}^{T} \mathbf{A}_{x x}, \\
& \frac{\partial\left(\mathbf{B}_{x y}^{T} T_{x y}^{(2)}\right)}{\partial \mathbf{e}}=\frac{\partial \mathbf{B}_{x y}^{T}}{\partial \mathbf{e}} T_{x y}^{(2)}+\mathbf{B}_{x y}^{T} \frac{\partial T_{x y}^{(2)}}{\partial \mathbf{e}}=\mathbf{H}^{T} \frac{\partial \mathbf{A}_{x y}^{T}}{\partial \mathbf{e}} T_{x y}^{(2)}+G \mathbf{B}_{x y}^{T} \mathbf{A}_{x y},
\end{aligned}
$$

we get

$$
\mathbf{K}_{T}=\mathbf{K}_{T, x x}+\mathbf{K}_{T, x y}+\mathbf{K}_{T q, x x}+\mathbf{K}_{T q, x y},
$$

and after introducing $d x=\mathbf{J} d \xi$ and $\mathbf{J}=l / 2$, similarly as for the bar element 


$$
\begin{gathered}
\mathbf{K}_{T, x x}=E \int_{V} \mathbf{B}_{x x}^{T} \mathbf{B}_{x x} d V=E \int_{V} \mathbf{H}^{T} \mathbf{A}_{x x}^{T} \mathbf{A}_{x x} \mathbf{H} d V=E \frac{l}{2} \int_{-1}^{1} \int_{A} \mathbf{H}^{T}(\xi) \mathbf{A}_{x x}^{T}(\xi) \mathbf{A}_{x x}(\xi) \mathbf{H}(\xi) d A d \xi, \\
\mathbf{K}_{T, x y}=G \int_{V} \mathbf{B}_{x y}^{T} \mathbf{B}_{x y} d V=G \int_{V} \mathbf{H}^{T} \mathbf{A}_{x y}^{T} \mathbf{A}_{x y} \mathbf{H} d V=G \frac{l}{2} \int_{-1 A}^{1} \int_{A} \mathbf{H}^{T}(\xi) \mathbf{A}_{x y}^{T}(\xi) \mathbf{A}_{x y}(\xi) \mathbf{H}(\xi) d A d \xi, \\
\mathbf{K}_{T q, x x}=\int_{V} \mathbf{H}^{T} \mathbf{F}_{x x} T_{x x}^{(2)} \mathbf{H} d V=\frac{l}{2} \int_{-1 A}^{1} \int_{A} \mathbf{H}^{T}(\xi) \mathbf{F}_{x x}(\xi) T_{x x}^{(2)}(\xi) \mathbf{H}(\xi) d A d \xi \\
\mathbf{K}_{T q, x y}=\int_{V} \mathbf{H}^{T} \mathbf{F}_{x y} T_{x y}^{(2)} \mathbf{H} d V=\frac{l}{2} \int_{-1 A}^{1} \int_{A} \mathbf{H}^{T}(\xi) \mathbf{F}_{x y}(\xi) T_{x y}^{(2)}(\xi) \mathbf{H}(\xi) d A d \xi .
\end{gathered}
$$

In the case of large rotations $\mathbf{F}_{x x}=\partial\left(\mathbf{A}_{x x}^{T}\right) / \partial \mathbf{e}$

$$
\mathbf{F}_{x x}=\left[\begin{array}{cccc}
1 & & s y m \\
0 & 1 & & \\
-y \cos \left(\varphi_{0}+\varphi\right) & -y \sin \left(\varphi_{0}+\varphi\right) & y^{2} & \\
y\left(\varphi_{0 . x}+\varphi_{. x}\right) \sin \left(\varphi_{0}+\varphi\right) & -y\left(\varphi_{0 . x}+\varphi_{. x}\right) \cos \left(\varphi_{0}+\varphi\right) & F_{x x 43} & F_{x x 44}
\end{array}\right],
$$

where

$$
\begin{gathered}
F_{x x 43}=y\left[\left(1+u_{0 . x}+u_{. x}\right) \sin \left(\varphi_{0}+\varphi\right)-\left(v_{0 . x}+v_{. x}\right) \cos \left(\varphi_{0}+\varphi\right)\right], \\
F_{x x 44}=y\left(\varphi_{0 . x}+\varphi_{. x}\right)\left[\left(1+u_{0 . x}+u_{. x}\right) \cos \left(\varphi_{0}+\varphi\right)+\left(v_{0 . x}+v_{. x}\right) \sin \left(\varphi_{0}+\varphi\right)\right] \\
\mathbf{F}_{x y}=\frac{\partial\left(\mathbf{A}_{x y}^{T}\right)}{\partial \mathbf{e}}=\left[\begin{array}{cccc}
0 & s y m \\
0 & 0 & \\
0 & 0 & 0 & \\
-\cos \left(\varphi_{0}+\varphi\right) & -\sin \left(\varphi_{0}+\varphi\right) & 0 & F_{x y 44}
\end{array}\right],
\end{gathered}
$$

where

$$
F_{x y 44}=\left(1+u_{0 . x}+u_{. x}\right) \sin \left(\varphi_{0}+\varphi\right)-\left(v_{0 . x}+v_{. x}\right) \cos \left(\varphi_{0}+\varphi\right) .
$$

In the case of small rotations

$$
\mathbf{F}_{x x}=\left[\begin{array}{cccc}
1 & 0 & -y & 0 \\
0 & 1 & -y \varphi_{0} & -y \varphi_{0 . x} \\
-y & -y \varphi_{0} & y^{2}\left(1+\varphi_{0}^{2}\right) & y^{2} \varphi_{0 . x} \varphi_{0} \\
0 & -y \varphi_{0 . x} & y^{2} \varphi_{0 . x} \varphi_{0} & y^{2} \varphi_{0 . x}^{2}
\end{array}\right], \mathbf{F}_{x y}=\left[\begin{array}{cccc}
0 & 0 & 0 & -1 \\
0 & 0 & 0 & -\varphi_{0} \\
0 & 0 & 0 & y\left(1+\varphi_{0}^{2}\right) \\
-1 & -\varphi_{0} & y\left(1+\varphi_{0}^{2}\right) & 2 y \varphi_{0 . x} \varphi_{0}
\end{array}\right] .
$$


Applying Newton-Raphson's method, in the $i$-th step we calculate - see i.e. Rakowski [11]:

- the tangent matrix $\mathbf{K}_{T}\left(\mathbf{q}^{(i-1)}, \mathbf{q}_{0}\right)$,

- the increment of displacements $\Delta \mathbf{q}^{(i)}=\mathbf{K}_{T}^{-1}\left(\mathbf{q}^{(i-1)}, \mathbf{q}_{0}\right) \Delta \mathbf{P}^{(i-1)}$,

- the displacements of nodes $\mathbf{q}^{(i)}=\mathbf{q}^{(i-1)}+\Delta \mathbf{q}^{(i)}$,

- the effect exerted by the element of the nodes $\mathbf{F}^{(i)}\left(\mathbf{q}^{(i)}, \mathbf{q}_{0}\right)=\int_{V}\left(\mathbf{B}_{x x}^{T} T_{x x}^{(2)}+\mathbf{B}_{x y}^{T} T_{x y}^{(2)}\right) d V$,

- unbalanced forces $\Delta \mathbf{P}^{(i)}\left(\mathbf{q}^{(i)}, \mathbf{q}_{0}\right)=\mathbf{P}-\mathbf{F}^{(i)}$.

The iteration is completed when $\Delta \mathbf{P}^{(i)} \leq \varepsilon$.

Expressing the second Piola-Kirchhoff stress tensor in the form of a matrix (2.10) and taking into account (4.2), the effects exerted by the elements on the nodes may be taken down as follows

$$
\mathbf{F}\left(\mathbf{q}, \mathbf{q}_{0}\right)=\mathbf{K}_{x x}\left(\mathbf{q}, \mathbf{q}_{0}\right) \mathbf{q}+\mathbf{K}_{x y}\left(\mathbf{q}, \mathbf{q}_{0}\right) \mathbf{q}+\mathbf{K}_{x x}^{0}\left(\mathbf{q}, \mathbf{q}_{0}\right) \mathbf{q}_{0}+\mathbf{K}_{x y}^{0}\left(\mathbf{q}, \mathbf{q}_{0}\right) \mathbf{q}_{0},
$$

where the components of the matrix of secants is calculated making use of the formulae

$$
\begin{aligned}
& \mathbf{K}_{x x}\left(\mathbf{q}, \mathbf{q}_{0}\right)=E \int_{V} \mathbf{H}^{T} \mathbf{A}_{x x}^{T} \mathbf{E}_{x x} \mathbf{H} d V=E \frac{l}{2} \int_{-1}^{1} \int_{A} \mathbf{H}^{T}(\xi) \mathbf{A}_{x x}^{T}(\xi) \mathbf{E}_{x x}(\xi) \mathbf{H}(\xi) d A d \xi, \\
& \mathbf{K}_{x y}\left(\mathbf{q}, \mathbf{q}_{0}\right)=G \int_{V} \mathbf{H}^{T} \mathbf{A}_{x y}^{T} \mathbf{E}_{x y} \mathbf{H} d V=G \frac{l}{2} \int_{-1 A}^{1} \int_{\mathbf{H}^{T}} \mathbf{H}^{T}(\xi) \mathbf{A}_{x y}^{T}(\xi) \mathbf{E}_{x y}(\xi) \mathbf{H}(\xi) d A d \xi, \\
& \mathbf{K}_{x x}^{0}\left(\mathbf{q}, \mathbf{q}_{0}\right)=E \int_{V} \mathbf{H}^{T} \mathbf{A}_{x x}^{T} \mathbf{E}_{x x}^{0} \mathbf{H} d V=E \frac{l}{2} \int_{-1 A}^{1} \int_{\mathbf{H}^{2}} \mathbf{H}^{T}(\xi) \mathbf{A}_{x x}^{T}(\xi) \mathbf{E}_{x x}^{0}(\xi) \mathbf{H}(\xi) d A d \xi, \\
& \mathbf{K}_{x y}^{0}\left(\mathbf{q}, \mathbf{q}_{0}\right)=G \int_{V} \mathbf{H}^{T} \mathbf{A}_{x y}^{T} \mathbf{E}_{x y}^{0} \mathbf{H} d V=G \frac{l}{2} \int_{-1 A}^{1} \int_{1} \mathbf{H}^{T}(\xi) \mathbf{A}_{x y}^{T}(\xi) \mathbf{E}_{x y}^{0}(\xi) \mathbf{H}(\xi) d A d \xi .
\end{aligned}
$$

The values of the integrals are calculated numerically by means of the Gauss quadrature, taking into consideration that

$$
\int_{A} d A=A, \int_{A} y d A=I_{z 1}, \int_{A} y^{2} d A=I_{z 2}, \int_{A} y^{3} d A=I_{z 3}, \int_{A} y^{4} d A=I_{z 4} .
$$

Denoting the product of the matrix below the integrals as $\mathbf{W}(\xi)$, after the application of Gauss's quadrature we get - see i.e. Majchrzak [23]

$$
\int_{-1}^{1} \mathbf{W}(\xi) d \xi=\sum_{i_{g}=1}^{n_{g}} \mathbf{W}\left(\xi_{i g}\right) \cdot w_{i g},
$$

where: $w_{i g}, \xi_{i g}$ - weights and abscissas of the Gauss points. 
The internal forces in the primary system, the co-called Kirchhoff forces, are defined similarly as in Mingrui Li [1]

$$
N=\int_{A} T_{x x}^{(2)} d A, V=\int_{A} T_{x y}^{(2)} d A, M=\int_{A} y T_{x x}^{(2)} d A .
$$

In the case of a transverse force it is assumed that $\int_{A} d A=\kappa A$, where $\kappa$ is the corrective factor, which takes into account the uneven distribution of tangential stresses on the surface of the cross-section area.

\section{EXAMPLES}

In the first example a bi-articulated steel arch, protected against buckling out of the plane, with a span of $10.0 \mathrm{~m}$ and a central angle of $120^{\circ}$, was analyzed, with characteristics of its cross-section $A=7,48 \mathrm{~cm}^{2}, I_{\mathrm{z}}=33,2 \mathrm{~cm}^{4}$. The geometry of the arch was determined by assuming a initial displacement of the respective nodes of the straight bar $A B$ and their initial rotation, dividing it into equal elements with two, three, five, seven, nine or eleven nodes. Two cases of loads were considered, viz. by a vertical force $P$ or horizontal force $H$ (Fig. 3). In the second case, at the load $H \neq 0$, the possibility of a shift of the support $B$ was assumed. The effect of the dead load was left out of account.

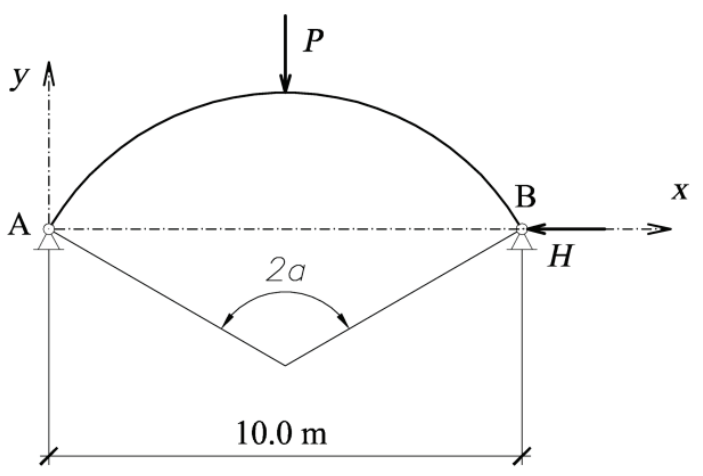

Fig. 3. Characteristics of the arch

The correctness of the solution dealt with in this paper, as well as the effect of dividing the load into the results of calculations was checked up on the example of an arch with a central angle of $120^{\circ}$, loaded with the force $P=30 \mathrm{kN}$. The arch was divided into 53 five-node elements, equal in their lengths, situated along the chord which connects the nodes $A$ and $B$. The load was divided into 5 steps. After each step, 
with growing values of the force $P$, the displacements of the nodes were recorded, and then the value of the load $P$ was reduced in five steps until $P=0 \mathrm{kN}$. The displacements of all the nodes in the steps of loading and unloading, corresponding to each other, were identical, and all the components of elastic displacements after the total unloading of the arch were of equal to zero.

The second test consisted in comparing the vertical displacements of the keystone of the arch with increasing different loads up to their final value. In every case, independently of sharing the load, the same values of displacement were obtained as in the case of a full load applied in one step of loading, which complies with the conclusions quoted by the author of [1].

Next, the effect of changing the number of elements $\left(n_{\mathrm{e}}\right)$ and the number of nodes $\left(n_{\mathrm{w}}\right)$ in the element on the process of iterating and the results of calculations were analyzed, when the number of the points of numerical integration $\left(n_{\mathrm{G}}\right)$ corresponded to the number of nodes in a single element. In the case of an element with five nodes additional calculations were carried out for 4,6 and 8 Gauss points. The results obtained at a load with the force $P=30 \mathrm{kN}$ in the form of a vertical displacement of the keystone node of the arch were gathered in column 5 of Table 1.

Table 1

Selected results of calculations an arch with a central angle of $120^{\circ}$, at $P=30 \mathrm{kN}$

\begin{tabular}{|c|c|c|c|c|c|c|c|}
\hline Lp. & $n_{\mathrm{e}}$ & $n_{\mathrm{w}}$ & $n_{\mathrm{G}}$ & $u_{\mathrm{y}}[\mathrm{mm}]$ & $\%$ & $n_{\mathrm{em}}$ & $n_{\mathrm{i}}$ \\
\hline 1 & 2 & 3 & 4 & 5 & 6 & 7 & 8 \\
\hline \multirow{3}{*}{1} & 150 & \multirow{3}{*}{2} & \multirow{3}{*}{2} & 303,34 & $-44,71$ & 2679 & 7 \\
\hline & 500 & & & 511,04 & $-6,85$ & 9879 & 10 \\
\hline & 1000 & & & 538,69 & $-1,81$ & 17979 & 10 \\
\hline \multirow{4}{*}{2} & 53 & \multirow{4}{*}{3} & \multirow{4}{*}{3} & 545,95 & $-0,48$ & 2817 & 10 \\
\hline & 103 & & & 548,38 & $-0,04$ & 5517 & 10 \\
\hline & 153 & & & 548,56 & $-0,01$ & 8217 & 10 \\
\hline & 253 & & & 548,60 & 0,00 & 13617 & 10 \\
\hline \multirow{6}{*}{3} & 8 & \multirow{3}{*}{5} & \multirow{3}{*}{5} & 548,07 & $-0,10$ & 1320 & 10 \\
\hline & 13 & & & 548,47 & $-0,02$ & 2220 & 10 \\
\hline & 18 & & & 548,60 & 0,00 & 3120 & 10 \\
\hline & 33 & 5 & 4 & 548,60 & 0,00 & 5820 & 10 \\
\hline & 33 & 5 & 6 & 548,60 & 0,00 & 5820 & 10 \\
\hline & 33 & 5 & 8 & 548,60 & 0,00 & 5820 & 10 \\
\hline \multirow{3}{*}{4} & 5 & \multirow{3}{*}{7} & \multirow{3}{*}{7} & 547,54 & $-0,19$ & 1659 & 10 \\
\hline & 8 & & & 548,61 & 0,00 & 2793 & 10 \\
\hline & 13 & & & 548,58 & 0,00 & 4683 & 10 \\
\hline
\end{tabular}


Table 1

\begin{tabular}{|c|c|c|c|c|c|c|c|}
\hline Lp. & $n_{\mathrm{e}}$ & $n_{\mathrm{w}}$ & $n_{\mathrm{G}}$ & $u_{\mathrm{y}}[\mathrm{mm}]$ & $\%$ & $n_{\mathrm{em}}$ & $n_{\mathrm{i}}$ \\
\hline \multirow{4}{*}{5} & 3 & \multirow{4}{*}{9} & \multirow{4}{*}{9} & 545,77 & $-0,52$ & 1566 & 10 \\
\hline & 5 & & & 548,40 & $-0,04$ & 2862 & 10 \\
\hline & 8 & & & 548,60 & 0,00 & 4806 & 10 \\
\hline & 13 & & & 548,59 & 0,00 & 8046 & 10 \\
\hline \multirow{3}{*}{6} & 3 & \multirow{3}{*}{11} & \multirow{3}{*}{11} & 547,85 & $-0,14$ & 2409 & 10 \\
\hline & 5 & & & 548,52 & $-0,01$ & 4389 & 10 \\
\hline & 8 & & & 548,60 & 0,00 & 7359 & 10 \\
\hline
\end{tabular}

Column 6 contains the proportional percentage-wise differences of this displacement in relation to the maximum displacement $u_{\mathrm{y}, \max }=548,60 \mathrm{~mm}$, obtained in the calculations. As a visible measure of the effectiveness of the iteration the number of elements of the matrix of rigidity of the arch was applied $\left(n_{\mathrm{em}}-\right.$ column 7$)$, because in each analyzed case the number of required steps of iteration $\left(n_{\mathrm{i}}\right)$ amounted, with only few exceptions, to 10 (c.f. column 8).

In the case of elements with two nodes and the division of the arch into 1000 elements the result of the displacement $u_{\mathrm{y}, \max }$ was lower than by $1,81 \%$. A more accurate value may be obtained by means of a still denser division. Herein a shear locking effect was to be observed. When three-node elements were applied, a good accuracy was achieved by dividing the bar into about 250 elements, and in the case of elements with five nodes a similar accuracy was achieved by dividing the bar into about 18 elements. In the case of elements with seven, nine and eleven nodes it is sufficient to divide them into 8 elements. Taking into account the results presented above, as well as the total number of elements in the stiffness matrix $\left(n_{\mathrm{em}}\right)$, it may be assumed that the most favorable elements for calculations of strongly deformed bars are elements with five and seven nodes.

Fig. 4 presents the displacement of an arch with a central angle of $120^{\circ}$, obtained at the vertical force $P=25 \mathrm{kN}$ and $P=50,0 \mathrm{kN}$, and a division of the bar into 18 five-node elements.

In the case of applying the force $P=25 \mathrm{kN}$ the preliminary deformation of the bar was introduced into the calculations in two ways, viz. with and without initial rotations. Due to the application of preliminary rotations the value of the deflection of the node in the keystone decreased from $628 \mathrm{~mm}$ to $395 \mathrm{~mm}$, i.e. by $37 \%$.

Fig. 5 contains the results of calculations concerning an arch loaded with the force $H=3 \mathrm{kN}$, similarly as in the former case in two variants - with and without preliminary rotations. In this case the horizontal displacement of the node $B$ decreased from $7,899 \mathrm{~m}$ to $7,692 \mathrm{~m}$, i.e. by $2,62 \%$. For example, the following characteristics of the crosssection were assumed: $A=6 \mathrm{~cm}^{2}, I_{\mathrm{z}}=18 \mathrm{~cm}^{4}$. 


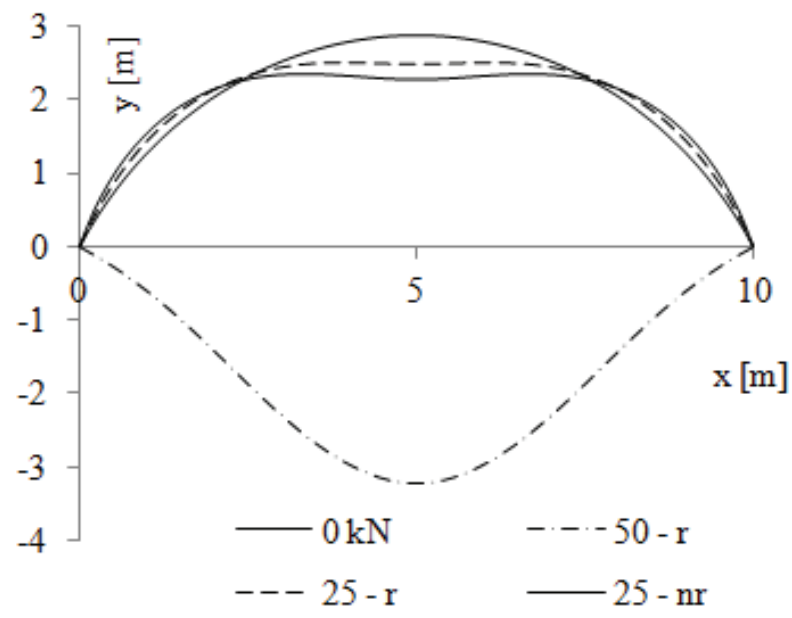

Fig. 4. Displacements of the axis of the arch loaded with $P=25 \mathrm{kN}$ and $\mathrm{P}=50 \mathrm{kN}$

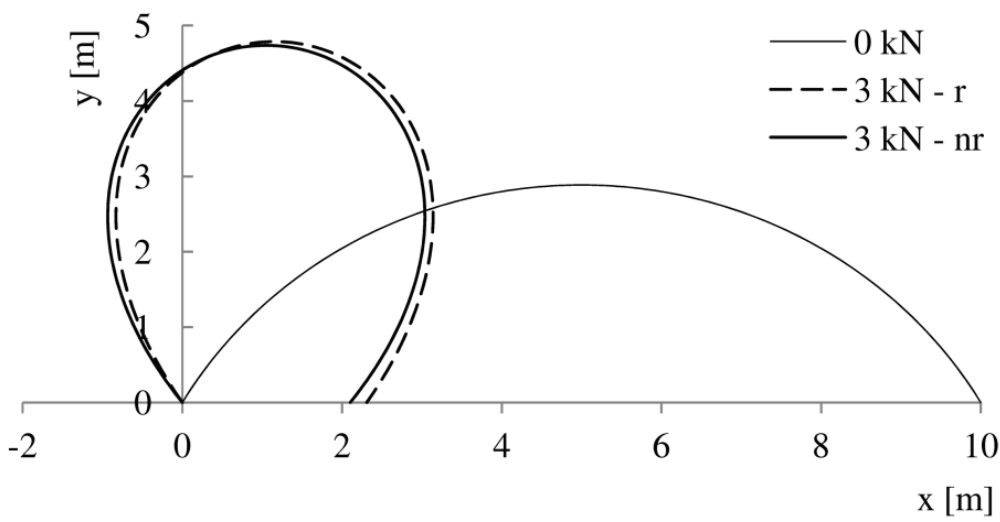

Fig. 5. An arch loaded in the node $B$ with the force $H=3 \mathrm{kN}$

In the second example a tie rod with a span of $12,0 \mathrm{~m}$ from a bar $\phi=20 \mathrm{~mm}$ with the initial sag $f_{0}=2,715 \mathrm{~m}$, loaded with the concentrated force $P=40 \mathrm{kN}$ in the centre of the span was analysed. The shape of the tie rod was obtained by initial deformations of its chord to the form of a chain curve by means of initial displacements and rotations of the nodes (fig. 6). The influence of the dead weight of the tie rod was left out of account. The solution was attained by dividing the bar into 18 elements with five nodes. The displacement of the central node amounted to $341 \mathrm{~mm}$. 


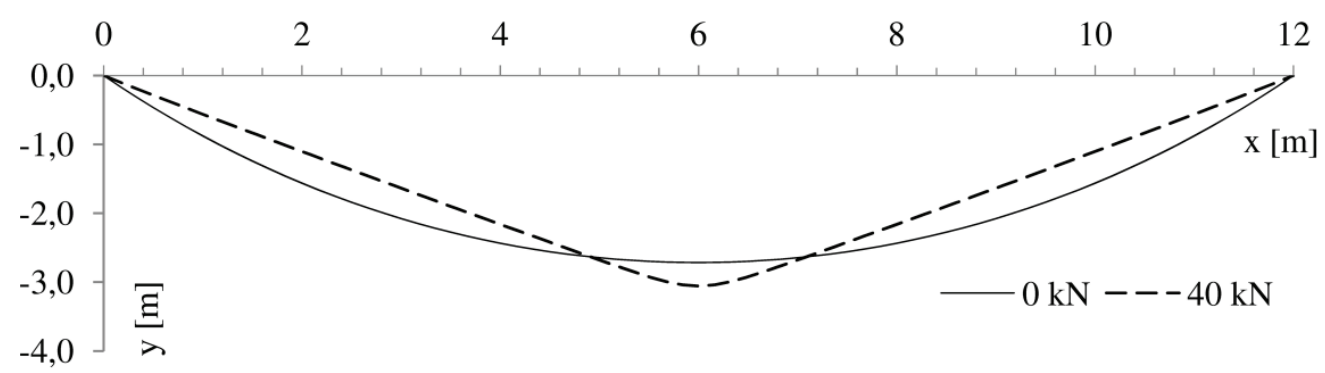

Fig. 6. Tie rod loaded in the centre of the span with force $P=40 \mathrm{kN}$

In the third ex ample, an initially deformed tubular bar $\left(A=7,48 \mathrm{~cm}^{2}, I_{\mathrm{z}}=33,2 \mathrm{~cm}^{4}\right)$ with a span of $5 \mathrm{~m}$ was analyzed, supported articulately in the node $A$ and in an articulated- movably way in the node $B$; the nodes being denoted as in Fig. 3. An initially deformation of the bar was assumed which might possibly occur in an actual structure, expressed by the function

$$
y_{0}(x)=\frac{l}{300} \sin \frac{\pi x}{l}+\frac{l}{600} \sin \frac{2 \pi x}{l} .
$$

The load was assumed to be the force $H$ and the moment $M$, imposed in the cross-section adjacent to the node $B$. The results of the calculations were gathered in Fig. 7a, concerning loads exerted by the axial force, and in Fig. 7b in the case of applying both the axial force and the bending moment.

a)

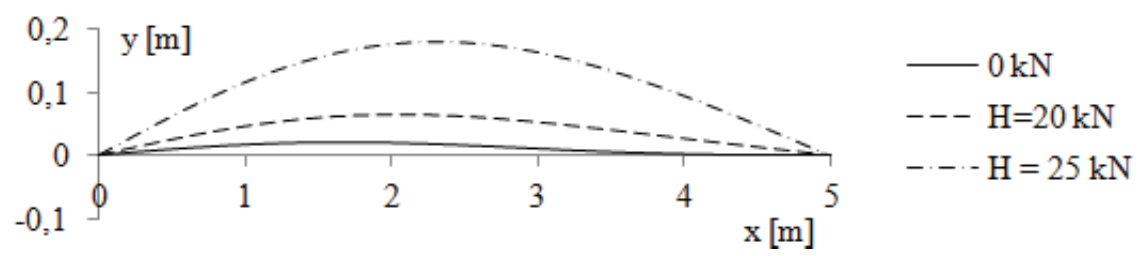

b)

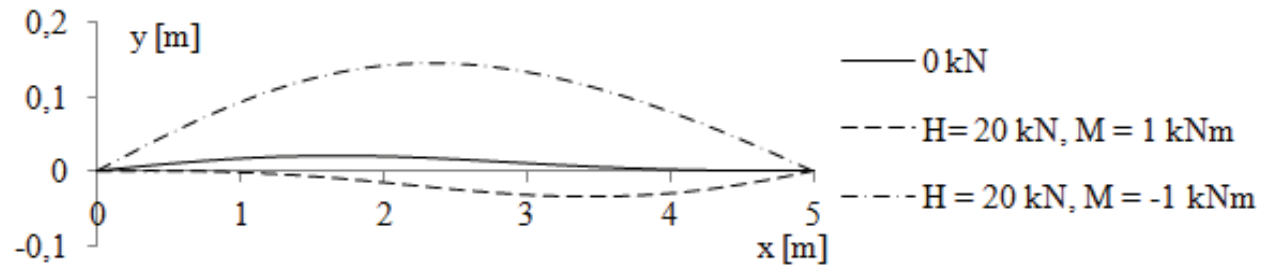

Fig. 7. Initially deformed bar loaded on the node with the force $H$ and moment $M$ :

a) load $H$, b) load $H$ and $M$ 


\section{Conclusion}

In the paper presents the FEM solution of an initially, stressless deformed frame element with an arbitrary field of preliminary deformations - shifts and rotations (e.g. of the arch or tie rod) with node points situated along the axis of an initially deformed bar are presented. The solution takes into account the effect of shear similar to that in the Timoshenko beam, the effect of large displacements (Green-Lagrange's strain tensor) and also moderately large rotations, i.e. such ones which permit an elastic behaviour of the material, and alternatively small rotations, applying the second Piola-Kirchhoff stress tensor. The whole Total Lagrangian description was applied. The displacements were approximated by means of higher-order polynomial shape functions. The presented examples contain elements with two, three, five, seven, nine and eleven nodes. Basing on calculations it was estimated that in the case of a large initial deformation it is expedient to use elements with five or seven nodes. In the case of two- and three node elements the convergence of the solution is very weak and weak. The bar must be divided into more than 1000 and about 250 elements, respectively.

The results of the solution are independent of the division of the load into incrementing steps. The final values of displacements are always the same.

The performed analysis also proved that the elastic deformation of the element depends not only on the effect of preliminary displacements of the nodes, but also on the effect of their rotations. These values may differ considerably.

The solution suggested in the paper can be applied in calculations of all kinds of flat initially deformed bars, protected against the loss of the stability in their plane. They may be both bars of a light steel structures and covers of various kinds of buildings, as well as elements of various column-beam structures. This solution may also be used to calculate flat steel arches as well as bent and stretched tension members, occurring both general building engineering (like sports and exhibition halls) and bridges. It may also prove to be applicable in assessment of the accuracy of other, less exact calculations based, for instance, on the theory of the second order.

\section{REFERENCES}

1. Mingrui Li, The finite deformation theory for beam, plate and shell Part I. The two-dimensional beam theory, Comput. Methods Appl. Mech. Engrg. Elsevier, Volume 146, Issues 1-2, 53-63, 5 July 1997.

2. K.J. Bathe, Finite Element Procedures. Prentice-Hall Inc., Simon \& Schuster/A Viacom Company, Upper Saddle River, New Jersey 07458, 1996.

3. A. Borkowski, Cz Cichoń, M. Radwańska, A. Sawczuk, Z. Waszczyszyn, Structural mechanics, Computer formulation (in Polish), T.3, Arkady, Warszawa 1995.

4. J. E. F. Guimaraes, G. R. Heppler, On trigonometric basis functions for $\mathrm{C} 1$ curved beam elements, Computers and Structures, 45, 405-413, 1992.

5. A. Ibrahimbegovic, On finite element implementation of geometrically nonlinear Reissner's beam theory: three-dimensional curved beam elements, Computer Methods in Applied Mechanics and Engineering, 122 11-26, 1995. 
6. G. Jelenić, M.A. Crisfield, Geometrically exact 3D beam theory: implementation of a strain-invariant finite element for statics and dynamics, Computer Methods in Applied Mechanics and Engineering, Volume 171, Issues 1-2, 141-171, 26 March 1999.

7. M. B. Kanchi, Matrix Methods of Structural Analysis, Second and enlarged edition, John Wiley \& Sons, 1993.

8. P. Litewka, Effective finite element with a large curvature (doctor's dissertation in Polish), Politechnika Poznańska IKB, 1998.

9. S. Lenci, F. Clementi, Simple Mechanical Model of Curved Beams by a 3D Approach Journal of Engineering Mechanics135, 597, 2009.

10. P. F. Pai, A., N. Palazotto, Large-deformation analysis of flexible beams. International Journal of Solids and Structures, Volume 33, Issue 9, 1335-1353, April 1996.

11. G. Rakowski G, Z. Kacprzyk, Finite element method in structural mechanics (in Polish), Oficyna Wydawnicza Politechniki Warszawskiej, Warszawa 1993.

12. A. Rosen, O. Gur, A transfer matrix model of large deformations of curved rods, Journal Computers and Structures, Volume 87, Issue 7-8, 467-484, April 2009.

13. S. Tang, A. Yu, Generalized variational principle on nonlinear theory of naturally curved and twisted beams, Applied Mathematics and Computation, Volume 153, Issue 1, 275-288, 25 May 2004.

14. O. C. Zienkiewicz, R. L. Taylor, The Finite Element Method, Fifth edition, Volumene 2: Solid Mechanics, Butterworth Heinemann 2000.

15. Z. Kączkowski, G. Rakowski, Z. Waszczyszyn, Polish structural mechanics at the turn of the 20th century, Archives of Civil Engineering, LI, 4, 2005, 431-470.

16. J. Gołaś, The influence of shear deformation on the transversal vibrations of viscoelastic fibrous composite beam, Archives of Civil Engineering, LIII, 2, 2007, 211-224.

17. J. Górski, Simulation-based nonlinear analysis of imperfect structures, Archives of Civil Engineering Volume 47, 1, 2007, 3-18.

18. J. Zamorowski, Preliminarily deformed flat bar element. A comparison of solutions (in Polish), XII Międzynarodowa Konferencja Naukowo-Techniczna „Konstrukcje Metalowe”, 404-413, Wrocław 15-17 czerwca $2011 \mathrm{r}$.

19. R. Adman, H. Afra, Advances in Engineering Software, Volume 38, Issues 8-9, 576-585, AugustSeptember 2007.

20. M. Kleiber, Engineering Mechanics. Computer methods of mechanics of solids (in Polish), t. XI, PWN Warszawa 1995.

21. M. Paluch, Fundamentals of the mechanics of deformable media (in Polish), Wydanie II rozszerzone, CIT Kraków 1997.

22. C. A. Felippa, B. Haugen, Unified Formulation of Small-Strain Corotational Finite Elements: I. Theory. Report No. CU-CAS-05-02 Contributed to Computer Methods in Applied Mechanics and Engineering for the Special Issue on Shells, edited by E. Ramm, M. Papadrakakis and W. A. Wall. 2005.

23. E. Majchrzak, B. Mochnacki, Numerical methods. Basic theoretical, practical aspects and the algorithms (in Polish), Wydawnictwo Politechniki Śląskiej, Gliwice 2004. 